\title{
Light and Chemical Energy ${ }^{*}$
}

\section{Perrin's Brilliant New Theory with Regard to Effect of Wave Length Upon Reactions}

\author{
By Professor Pierre Weiss, of the University of Strasbourg
}

$\mathrm{L}$ ET us now consider the question of polarized light. As we know luminous vibrations are transverse instead of being longitudinal like those of sound. A metaphor will readily enable us to understand what is meant by the polarization of light. It is a simple matter to put a letter in the mail box if we present its edge parallel to the slit in the box, but if we present it in a perpendicular position to the slit it will not enter. The apparatus known as a pularizer may be compared to a letter box having a vertical slit. Let us now suppose a second slit in the letter box opposite the first. If these two slits are perpendicular to each other the letter will not pass through; if they are parallel it will pass. Correspondingly we may take a second polarizer and place it opposite the first in the path of a ray of light. To distinguish the two we call this second polarizer the analyzer, a word which expresses its function. The first apparatus is placed so as to allow vertical vibrations to pasis. If the analyzer is arranged in the same way light will pass through the two without any difficulty, just as the letter passes through the two parallel slits in the box. But if we cause the second apparatus to revolve $90^{\circ}$ around the ray of light as an axis of rotation, in this position it will allow only horizontal light to pass, and since the light coming from the polarizer is vertical no light will be able to pass.

Now let us make an experiment. By the rotation of the analyzer at a right angle we pass from full light to darkness, i.e., extinction and then by a new rotation, likewise of $90^{\circ}$, we pass from extinction to full light and so forth. It is obvious that this experiment can be comprehended only by supposing that light has a transverse vibration, since if light were moving in the same direction as the ray there would be no change.

Let us now interpose between the polarizer and the analyzer in the extinction position a sheet of a crystallized substance, e.g., a sheet of gypsum having an unequal thickness between one end and the other and obtained by the cleavage of a crystal of gypsum. Crystallized matter possesses the property of causing a profound disturbance in the direction of the vibrations of light which it receives. The vertical vibration falling upon it will be rendered partly horizontal, thus allowing light to pass. But since this effect is unequal in extent in the case of light rays of different colors, very vivid colors will frequently make their appearance. Thus we shall see certain areas of the gypsum appear upon the screen as red, others as green, and still others as yellow. Each thickness of the gypsum will give a different mixture of colors.

Now if we turn the analyzer at a right angle the light which passed in the previous experiment will be arrested and vice versa . . . so that in the second part of the experiment we shall obtain the colors which are the exact complement of those in the first, since the ensemble produced white light.

Instead of abserving these two aspects of the light successively we can obtain them simultaneously side by side upon the screen, in which case we see clearly that a green area in one corresponds to a red area in the other and so on.

*Translated for the Scientific American Monthly from an address made June 2, 1920, at the Congress of the Union of the Industrial Societies of France, and published in the Bulletin de la société Industrielle, Mulhouse (France), for June-July-August, 1920.
I have here several objects made from another crystalline substance - mica - such as the petals of a flower and the wings of a butterfiy. These are formed of sheets of mica of different thicknesses; when we place them between the polarizer and analyzer we see the image of the butterfiy or of the fiower appear in colors upon the screen, and when we throw them upon the screen side by side at the same time we shall have a butterfiy and a fiower tinted in complementary colors.

The phenomena due to the transverse nature of vibrations and the possibility of separating these int different directions around the ray of light are very various in nature. Instead of obtaining the re-appearance of the light by placing between the polarizer and the analyzer a sheet of crystalline substance, we may arrive at an analogous result by using a parallelepiped of ordinary glass. We can accomplish this by imparting to the glass artificially optical properties which vary with the direction. This is done by exerting strong pressure upon the glass in which case the compressed glass acts like a crystal and the direction of the pressure exerted corresponds to one of the axes of this crystal. And we find that the stronger the pressure exerted upon the glass the more intense the light which reappears. And by studying this phenomenon mathematically we can even deduce the exact distribution of the internal tensions of which the substance is the seat. And it has been found possible to find an optical solution of delicate problems with respect to the resistance exhibited by various materials, by reproducing in glass pieces of metallic framework which are difficult to calculate, and then exerting upon the glass tensions or stresses identical with those to which the pieces of metal are subjected."

These tensions are also encountered in tempered glasses, i.e., glasses which have been heated to a red glow and then cooled with sufficient abruptness to cause the surface to regain its rigidity first. We shall examine between the crossed analyzer and polarizer a series of such specimens of glass and we shall see that the very vivid play of colors observed gives a faithful image of the state of internal tension of the different areas.

Finally, we know that quartz crystals share with certain compounds of organic chemistry a certain property which has provided chemists with a key to the asymmetries of the molecule, namely; the power of rotation. But in the quartz this rotatory power which is manifested in full perfection only in the direction of the axis of the crystal, is a result of the crystalline structure. In the crystal of quartz the successive vibrations of the light are arranged like the steps of a winding staircase. If we interpose a plate cut from a crystal of quartz between the polarizer and the analyzer in the extinction position the rotation which it imparts to the vibrations will have the effect of producing a luminous movement which the analyzer will allow to pass. And since the rotation is unequal for the different parts of the spectrum the reestablished light will exhibit colors. Indeed, the colors due to the power of rotation are among the richest which we know how to produce. The artifice which we have already employed will give us a color and its complement at one and the same time and since it is

'See "Determining Stresses by Polarized Light" by George Weed Hall and Arthur L. Kimball, Jr., in Scientific American Monthly, January, 1921, pp. 49-53.-EDTOR. 
easy to superpose the two images we shall again see white light reconstituted by each couple of colored areas-blue and orange yellow, red and green, salmon and greenish blue.

If we observe the waves which traverse the surface of a sheet of water we perceive at once that there is a definite distance maintained between each two consecutive waves; this represents the length of the wave. With our crossed analyzer and polarizer we can perform one of the many experiments which enable us to determine the wave length of light. In the the rectangle of red light thrown upon the screen you observe a series of parallel bands which are alternately light and dark. We have divided the luminous movement into two portions. In the luminous band farthest to the right the two luminous movements arrive at the same time and reinforce each other. In the adjacent dark band one is half a wave-length behind the other, in consequence of which they counteract and destroy each other; a little farther in the second luminous band the retardation is twice as great, which gives us an entire wave and the movements once more added together, producing the full strength of light, then in the following band there is a retardation of $1 \frac{11}{2}$ wave lengths so that again the crest and trough of the waves meet and neutralize each other, and so on. . . By calculations based upon this phenomenon the wave length of the red light at the far end of the spectrum has been found to be eight ten thousandths of a millimeter.

Let us repeat this experiment performing it at the same time with red light and with green light, which we obtain by filtering white light through colored screens. Now let us place the two phenomena side by side-we observe that the red bands are farther apart than the green ones and perceive that the wave length of green light is shorter than that of red light. At the most highly refractive end of the spectrum the wave length is only four ten-thousandths of a millimeter. In the ultra-violet the length is still shorter while it is considerably longer in infra-red. The longest wave lengths of light which have been measured are one and one half tenths of a millimeter.

Let us suppose now that the temperature of a body is gradually raised. At a point slightly above $400^{\circ} \mathrm{C}$. the rays emitted will begin to make an impression upon the retina of our eye and the body will appear dark red, passing later through cherry red and orange until it reaches an increasingly dazzling white . . . The proportion of the various rays alters and the higher the temperature becomes the richer the light is, both absolutely and relatively in violet and ultra-violet rays. If we reverse the experiment by cooling the body we gradually reach the red light and, finally, this becomes too feeble to impress the retina-beyond this point we have the infra-red rays whose wave length is the longest known.

Now let us imagine a closed metal box in which a vacuum has been produced and which is then heated from the outside. The internal walls will become incandescent as the temperature increases and will emit light which will traverse the box at the prodigious velocity at which light travels, i.e., 300,$000 \mathrm{~km}$. per second, until the light from one wall reaches the opposite wall where it will be reflected or absorbed. We perceive, therefore, that it is not precisely correct to say that this box contains nothing; as a matter of fact it contains radiations moving in every direction. And these radiations consist of a mixture of lights of all the colors and of all the wave lengths in proportions depending upon the temperature of the box at any given moment. If the temperature is lower than $500^{\circ} \mathrm{C}$. there will be practically no visible rays present, but only infrared rays.

Now the same process of reasoning holds true if we imagine the box instead of containing a vacuum to contain a solid liquid or gaseous transparent medium and if we cool it to the ordinary temperature of the air which surrounds us. In other words this very room in which we are assembled represents such a box. It is evident, therefore, that we are as a matter of fact, immersed in infra-red rays which come from every direction to strike and penetrate our bodies. All the work which we do, every operation we perform in our daily lives takes place in a bath of infra-red rays. When we consider this evident fact it seems curious enough that no one has ever thought of assigning to this agent a rôle of importance in the phenomena which surround us.

\section{THE CHEMICAL ASPECT $\bullet$ THIS PHENOMENA}

Let us now examine the problem from a chemical point of view. Anyone familiar with chemical laboratories knows the important part played by heat in chemical processes. Whether he is concerned with one of the thousand operations of qualitative or quantitative analysis, whether he is dissolving a metal in an acid obtaining a precipitate or observing a color, the chemist nearly always heats the test tube in which the reaction takes place. A whole series of operations is conducted, by heating the bodies subjected to the experiment by means of a gas burner while they are traversing a tube.

Farly chemists made much use of furnaces fed by wood charcoal; now-a-days, we have coke furnaces or electric furnaces. . . such as those, for example, with which Moissan made his brilliant discoveries . . . It is a well-known fact that a rise of temperature accelerates chemical reactions, whereas a fall of temperature retards them. At the temperature of liquid air reactions which are violent at ordinary temperatures-such, for example, as that of sulphuric acid upon potassium-are benumbed. But it is an accepted fact that heat is due to molecular motion and that chemical combinations and decompositions result from the violent shocks produced between molecules which come in contact with each other because of this motion. But there are some difficulites in the way of accepting this theory.

When there is a rise of temperature to $10^{\circ} \mathrm{C}$. above the ordinary, the kinetic force of thermic agitation is augmented by a thirtieth and the velocity of the molecules by a fifteenth of their value. But there are a great many chemical reactions whose velocity increases much more rapidly-when it is even doubled, for example by a rise of ten degrees. It seems strange that there should be such a disproportion between cause and effect.

There is another difficulty which is perhaps even more serious. Let us suppose the case of a small quantity of the vapor of hydriodic acid (HI) in a closed container, say a liter. Let us now raise the temperature to such a point as to cause the vapor to break up into its component elements, hydrogen and iodine. This dissociation will take place at a certain rate of velocity.

Let us now put the same amount of vapor at the same temperature in a container holding 1000 liters. The molecules moving at the same velocity will have a thousand times as much space at their disposal and shocks of contact between them will be only $1 / 1000$ as frequent. And yet we know by experience that the dissociation takes place with the same velocity. Therefore, it would seem impossible that the dissociation is produced by the shocks of contact between molecules due to thermic agitation.

But these difficulties and discrepancies vanish if we assume that the reactions are occasioned, not by the thermic agitation itself, but by the accompanying radiation. Thus no matter what the size of the container at the same temperature the molecules of hydriodic acid would be immersed in the same medium, consisting of infra-red radiations-and, if the temperature is high enough, of visible and ultra-violet radiations also -rushing through space in every direction. If we could accept this theory therefore there is nothing to surprise us in the fact that the velocity of dissociation is independent of the space occupied by the substance.

\section{RELATION BETWEEN I.ENGTH OF WAVE AND CHEMICAL ACTION}

We must believe with Perrin that every chemical reaction is produced by a radiation having a specific wave-length. If this be true it would suffice, in order to estimate the variation in velocity of the reaction due to temperature, to know the vari- 
ation in the amount of this active light within the complex mixture of radiation. But it is a very remarkable fact that this calculation leads us to precisely that law which Arrhenius deduced from experiment some 30 years ago and which has hitherto remained inexplicable.

Inversely if we determine by experiment the velocity of the variation in a reaction due to temperature we can determine the wave-length of the ray of light which causes the reaction. For this purpose it is only necessary to choose among the complex mixture of rays of light of different wave-lengths that which has the same velocity of variation. Much study along this line will doubtless presently be undertaken-we will merely say now that in those cases in which such a calculation has thus far been made the wave-lengths obtained have been entirely plausible.

Let us now examine the same question from a different point of view. We know that colored substances merely select certain rays from white light and reflect them, while they absorb and destroy all the others. But there is one very important exception to this rule. Let us take a substance termed fluorescent, e.g., a piece of glass containing certain amounts of salts of uranium incorporated with it. Expose this to a beam of light containing only the violet light which we obtain by passing a beam of white light through a colored filter. In this violet beam the uranium glass will shine brilliantly diffusing in every direction a vivid greenish yellow light. Thus we see that uranium glass is capable of transforming the original violet light into yellow light, i.e., a light different both in color and in wave-length.

Organic chemistry provides numerous examples of fluorescent substances among which some of the best known are fluorescein, eosein, esculin, quinine sulphate, etc. The phenomen $\bullet$ is often intense enough to be perceptible even when the fluorescent substance is present only in an exceedingly dilute form or in extremely thin sheets. M. Ferrin placed under a microscope an extremely thin layer of a solution of these substances and exposed it to an exceptionally strong light by projecting upon it the image of the electric arc light. He was thus enabled to make an important and fruitful observation, namely: that the fluorescence of that part of the substance touched by the image of the arc light is extinguished at the end of a few minutes. If the preparation is so placed as to expose fresh portions of the substance to the light, these portions will become fluorescent and then be extinguished in their turn. It is evident, therefore, that fluorescence is not, as had been thought, a permanent quality of certain molecules, but is, on the contrary, the visible sign of their disappearance. The molecule struck by a certain radiation, by violet light, for example, dies as it projects a flash of green light and gives birth to a molecule of a different kind. The reason why the destruction of the substance at the moment it becomes fluorescent had hitherto been unperceived, is that until Perrin made his experiment investigators had always made use of too feeble an illumination and had employed such large quantities of the substance that the diminution of the latter could not be observed.

M. Perrin looks upon fluorescence as the typical case: of all chemical reaction: a radiation is absorbed and occasions the reaction which gives rise to the emission of another radiation. 'The peculiarity of fluorescent substances consists in the fact that the radiation emitte and in many cases also the radiation absorbed are found in the visible spectrum. But when we remember that the invisible spectrum is far more extensive than the visible spectrum including both the infra red and the ultra-violet rays, there is nothing surprising in the idea that the eye fails to perceive any radiation in those reactions which are the most numerous and most ordinary of all. M. Perrin has gone a step further deducing by the doctrine of quanta (which there is no need of going into here) from a knowledge of the wave-lengths of the exciting light and the emitted light the amount of heat liberated in a given chemical reaction. The calculation made by him concerns the transfor- mation of ozone into oxygen and the figures obtained were 62,000 calories, whereas 60,000 calories are obtained by direct observation. This is, very evidently, remarkably close agreement.

Let us take another experiment:

Let us expose to daylight some calcium sulphide containing traces of metallic impurities, and afterward examine this substance in a dark room. At first it shines with a comparatively bright light, but the intensity of the light diminishes by degrees. This is the phenomenon known as phosphorescence. The substance has absorbed certain rays of the light to which it has been exposed and later it yields these up. While this phenomenon is somewhat akin to fluorescence, it is distinguished from it by the fact that in reality it comprises two inverse reactions.

When the substance is exposed to sunlight some rays present in the incident light causes a chemical reaction of unknown nature and this reaction is accompanied by the emission of a certain infra-red ray. An exactly inverse reaction is involved necessarily in the re-emission of the absorbed light and the absorption of the emitted light. But in the mixture of infrared rays in which all substances are immersed at ordinary temperatures, the substance will find the precise radiation which it emitted to begin with. This radiation will incite the inverse reaction and as a result, the incident light will be emitted.

This theory suggests a method of verification. If it be true that it is a certain infra-red ray which causes the emission of the stored up light, then it ought to be possible to increase the effect by increasing the intensity of this infra-red light. This can be done by exposing the phosphorescent substance to the infra-red spectrum of an incandescent body. As a matter of fact this experiment was actually made by $\mathrm{E}$. Becquerel a good many years ago, but the results remained unexplained until it was found that they accord with Perrin's theory. When such exposure is made we first observe a more intense emission at those points where certain infra-red rays of a suitable wave-length fall upon the substance, but in these same areas the phosphorescence is more quickly extinguished so that they quickly become black, in contrast to those parts of the preparation which have not been exposed to the infra red ray.

Another manner of augmenting the intensity of the infrared consists in raising the temperature. Behind a stream of calcium sulphide which has been previously exposed to sunlight we placed a rectangular plate of sheet iron heated to about $100^{\circ} \mathrm{C}$. That area of the screen opposite the sheet iron becomes far brighter than the neighboring areas. But after a very short time their stored up light is exhausted and they become black.

The development of M. Perrin's theory promises to be of great value in science. If these new ideas are correct and it must be admitted that these primary verifications recited by us constitute a strong presumption in their favor, it is impossible to doubt that the knowledge thus gained of the actual mechanism concerned in chemical reaction must have a profound influence upon the development of both pure and applied chemical science.

We can readily imagine, indeed, a state of development of our chemical knowledge in some future day when, instead of making a crude use of the entire spectrum of radiations yielded by a given temperature, in which case certain ones of these rays produce the desired reaction, while others, on the contrary, produce the opposite reaction so as to partially. cancel the effect, while still others produce what may be termed parasitic reactions-instead of this clumsy method let me repeat, we shall know how to make a definite choice of the precise ray required to produce the given effect. And let us make a solemn vow that in the future French. science shall no longer be content merely with originating new theories and methods but shall determine to reap. her fitting share of the harvest whese seeds she has sown. 